\title{
Association between serum vitamin D deficiency and knee osteoarthritis
}

\author{
Behzad Heidari • Parham Heidari • \\ Karaim Hajian-Tilaki
}

Received: 21 October 2010 /Accepted: 2 December 2010 /Published online: 30 December 2010

(C) Springer-Verlag 2010

\begin{abstract}
Background Insufficient levels of serum 25-hydroxyvitamin D (25-OHD) influence the knee joint cartilage and lead to development and progression of knee osteoarthritis (OA). The purpose of this study was to determine the status of serum 25-OHD levels in patients with knee OA compared with controls.

Methods A total of 148 patients with knee OA and 150 controls were studied. Serum 25-OHD was measured by the ELISA method and concentrations $<20 \mathrm{ng} / \mathrm{ml}$ were considered as deficient levels. Mann-Whitney $U$ test was used for comparisons of means and logistic regression analysis with calculation of odds ratio (OR) was applied to determine association.

Results The mean ages of patients and controls were $60.2 \pm$ 12.9 and $60.1 \pm 10.2$ years, respectively $(P=0.23)$. In the entire population the mean serum 25-OHD in OA patients was not significantly lower than controls $(P=0.28)$, but in subgroup analysis the mean 25-OHD in OA patients aged $<60$ years was significantly lower than controls $(23.8 \pm 18.8$ vs. $34.5 . \pm 29.6 \mathrm{ng} / \mathrm{ml}, P=0.01)$. In this age group knee OA was significantly associated with serum
\end{abstract}

\author{
B. Heidari $(\triangle)$ \\ Department of Medicine, Division of Rheumatology, Rouhani \\ Hospital, Babol University of Medical Sciences, \\ Babol, Iran \\ e-mail: heidaribeh@yahoo.com \\ P. Heidari \\ Azad Islamic University of Karaj, \\ Karaj, Iran \\ K. Hajian-Tilaki \\ Department of Social Medicine, \\ Babol University of Medical Sciences, \\ Babol, Iran
}

25-OHD deficiency which remained significant after adjusting for age and sex (adjusted $\mathrm{OR}=2.26,95 \% \mathrm{CI}$ 1.15-4.4, $P=0.018$ ). A greater association was observed in patients aged $<55$ years $(\mathrm{OR}=2.63,95 \%$ CI $1.16-5.95, P=$ 0.01 ); whereas the association between $\mathrm{OA}$ and serum 25 OHD deficiency in patients aged $\geq 60$ years did not reach a significant level.

Conclusion These findings indicate a significant association between serum 25-OHD deficiency and knee OA in patients aged $<60$ years and suggest serum 25-OHD measurement in any patient with symptoms suggestive of knee OA particularly at the initial stage of disease.

\section{Introduction}

Vitamin D deficiency is a common problem worldwide [1, 2]. While sufficient levels of serum vitamin $D$ decrease the risk of many chronic illnesses, its inadequacy is associated with multiple pathological conditions including osteoarthritis $(\mathrm{OA})[3,4]$. Vitamin D status influences the incidence and progression of knee OA [5]. Sunlight exposure and serum 25-OHD levels are both associated with decreased knee cartilage loss [6]. In serum 25-OHD deficient men the prevalence of OA was two times greater than those with sufficient levels [7]. In OA, changes in subchondral bone play an essential role in the onset and progression of cartilage lesions. In this condition bone resorption markers are higher and bone formation markers are lower compared with a control group [4, 8]. In progressive OA, bone metabolism and bone turnover are increased similar to that observed in patients with osteoporosis. Low serum 25OHD increases osteoblastic activity and bone turnover [1]. Raising serum 25-OHD to sufficient levels with supplemental vitamin $\mathrm{D}$ will decrease the rate of bone turn-over, 
suppress the PTH level, increase BMD and even decrease fracture risk in the elderly population $[8,9]$.

Both OA and vitamin D deficiency are age-dependent [10]. During aging, knee OA becomes more symptomatic and prevalent, vitamin D stores decline, and subclinical vitamin D deficiency or insufficiency occurs in the elderly, especially in women [10]. This process may alter bone mineral metabolism and lead to progression of knee OA through development of osteopenia or exacerbation of osteoporosis [8]. A higher than expected rate of vitamin D deficiency, as has been reported in OA, indicates that these patients are at higher risk of disease progression [11, 12]. Hence, knowledge of the serum status of 25-OHD may provide additional information for recognising patients at risk of progression. To date, few studies have addressed this topic so data on this subject are lacking. We therefore performed this study to determine the status of serum vitamin $\mathrm{D}$ levels in patients with knee OA compared to agematched controls.

\section{Methods}

The population of this cross-sectional study consisted of patients with knee OA and age-matched controls. Patients were selected consecutively among individuals who presented with knee pain to an outpatient medical clinic located in Babol, Iran, a temperate geographic region at a latitude of $36^{\circ}$ on the Caspian Sea. Diagnosis of knee OA was confirmed by the American College of Rheumatology diagnostic criteria for classification of knee OA [13]. Sample size was determined with a confidence level of $95 \%$ and power of $80 \%$ to detect $15 \%$ difference in proportion of vitamin D deficiency between patients and controls at a significance level of $0.05 \%$. Considering a $30 \%$ vitamin D deficiency among the general population of this study [3], 146 subjects were required for each group. Subjects of the control group were selected among subjects who presented to the same clinic over the same period for nonskeletal symptoms such as recent respiratory or gastrointestinal symptoms or among subjects without any clinical symptoms who presented for check-up laboratory tests. The control group had no clinical features of knee OA based on history and clinical examination.

Serum 25-OHD was measured by ELISA method using a lyophilised competitive protein binding assay kit (DRG Instruments, $\mathrm{GmbH}$, Germany) according to instructions of the manufacturer. Serum 25-OHD levels less than $20 \mathrm{ng} / \mathrm{ml}$ were considered as deficient.

Exclusion criteria were presence of rheumatic diseases other than OA, chronic diseases of gastrointestinal, kidney and pulmonary systems, systemic debilitating diseases, limitation of physical activities, being on diet, supplemental vitamins and anticonvulsant drugs. Patients with severe radiographic knee OA at stage 4 Kelegren-Lawrence stage and those with knee joint instabilities were not included. Only patients with stable physical activities over the past three-month period were included. In statistical analysis the mean serum 25-OHD levels and proportion of serum 25OHD deficiency were determined in groups and compared with the matched controls. Additional subgroup analyses were performed according to age particularly age groups of less than 60 years versus $\geq 60$ years. Mann-Whitney U test was used for comparison of means. The association of vitamin $\mathrm{D}$ deficiency and knee OA was assessed using chisquare test with calculation of odds ratio (OR) and its $95 \%$ confidence interval $(95 \% \mathrm{CI})$. The independent effect of serum 25-OHD deficiency was determined after adjustment for sex and age using logistic regression analysis. SPSS software version 17 was used for analysis.

\section{Results}

A total of 148 patients with mean $( \pm \mathrm{SD})$ age of $60.2 \pm 12.9$, median of 60 (range 23-91) years old and 150 controls with mean $( \pm \mathrm{SD})$ age of $60.1 \pm 10.2$, median of 59 (range 36-85) years old $(P=0.23)$ entered the study. Serum 25-OHD deficiency was observed in $39.8 \%$ of patients versus $32 \%$ of controls $(P=0.18)$ (Table 1).

In the entire population of OA, the mean serum 25O-HD level in patients was lower than controls but the difference was not statistically significant $(34.1 \pm 32.4$ vs. $38.5 \pm$ $34.8 \mathrm{ng} / \mathrm{m}, P=0.28)$. There was an association between serum 25-OHD deficiency and knee OA which was not statistically significant after adjustment for gender (OR $1.62,95 \%$ CI $0.97-2.6, P=0.06$ ).

In the entire study population serum 25-OHD deficiency was not homogenously distributed across age but was more frequent in subjects aged $<60$ years compared with $\geq 60$ years particularly in the patient group (Table 1).

In subgroup analysis of subjects $<60$ years the mean serum 25-OHD level in patients was significantly lower $(23.8 \pm 18.8$ vs. $34.5 \pm 29.6 \mathrm{ng} / \mathrm{ml}, P=0.01)$ and proportion of serum 25-OHD deficiency was significantly higher than controls $(53.4 \%$ vs. $35.5 \%$, OR $=2.08,95 \%$ CI $1.07-4, P=$ 0.041 ). Whereas in populations aged 60 years and older there were no significant differences in means and proportions of serum 25-OHD deficiency between patients and controls $(44.1 \pm 39.2$ vs. $42.8 \pm 39.2 \mathrm{ng} / \mathrm{ml}, P=0.58$ and $26.6 \%$ vs. $28.3 \% ; P=1$, respectively).

In this age group there was a significant positive association between serum 25-OHD deficiency and knee OA which remained significant after adjusting for sex and age using logistic regression analysis $(\mathrm{OR}=2.26,95 \% \mathrm{CI}$ $1.15-4.4, P=0.018)$; whereas there was no association in 
Table 1 Mean serum 25-hydroxyvitamin D (25-OHD) and proportion of serum 25-OHD deficiency ${ }^{\mathrm{a}}$ in patients with knee osteoarthritis (OA) compared $^{\mathrm{b}}$ with controls according to age

\begin{tabular}{|c|c|c|c|}
\hline Serum 25-OHD & $\begin{array}{l}\text { All OA }(n=148) \text { vs. controls } \\
(n=150)\end{array}$ & $\begin{array}{l}<60 \text { years OA }(n=73) \text { vs. controls } \\
(n=76)\end{array}$ & $\begin{array}{l}\geq 60 \text { years OA }(n=75) \text { vs. controls } \\
(n=74)\end{array}$ \\
\hline Mean serum 25-OHD (ng/ml) & $34.1 \pm 32.4$ vs. $38.5 \pm 32.4 P=0.28$ & $23.8 \pm 18.8$ vs. $34.5 \pm 29.6 P=0.01$ & $44.1 \pm 39.2$ vs. $42.8 \pm 39.3 P=0.83$ \\
\hline Serum 25-OHD deficiency, $n(\%)$ & 59 (39.8) vs. 48 (32) $P=0.18$ & 39 (53.4) vs. 27 (35.5) $P=0.041$ & 20 (26.6) vs. 21 (28.3) $P=0.8$ \\
\hline OR $(95 \% \mathrm{CI})$ & $1.44(0.89-2.3)$ & $2.08(1.07-4)$ & $2.05(0.92-4.57)$ \\
\hline
\end{tabular}

$O A$ osteoarthritis, $O R$ odds ratio, $C I$ confidence interval

${ }^{\text {a }}$ Serum 25-OHD deficiency $<20 \mathrm{ng} / \mathrm{ml}$

${ }^{\mathrm{b}}$ Mann-Whitney U test and chi-square test were used for comparisons of means and proportions, respectively

age group of $\geq 60$ years old (Table 2). The association was greater in subjects aged less than 55 years $(\mathrm{OR}=2.63,95 \%$ CI 1.16-5.95, $P=0.01$ ).

Additional analysis in regard to mean serum 25-OHD and serum 25-OHD deficiency demonstrated a significant difference between means and the proportion of serum-25OHD deficiency according to age of less than 60 years compared with 60 years and older in the patient group but not in the control group $(23.8 \pm 18.8$ vs. $44.1 \pm 39.2 \mathrm{ng} / \mathrm{ml}, P=$ 0.001 , and $53.4 \%$ vs. $26.6 \%$, OR $=3.15,95 \%$ CI $1.58-6.27$, $P=0.001$, respectively).

\section{Discussion}

The findings of our study indicated a high prevalence rate of serum 25-OHD deficiency and a significant positive association between serum 25-OHD deficiency and knee OA in a subgroup of patients with knee OA aged less than 60 years with greater association in younger patients.

Up to now most studies addressed the association between OA and serum 25-OHD in longitudinal studies [5, 6, 14-17]. No study has yet compared serum 25-OHD levels between OA patients and controls. The results of this study are consistent with previous reports $[5-7,11,14,16]$ and add new information to current knowledge in relation to the association between serum 25-OHD deficiency and knee OA.

In the "Osteoporotic Fractures in Men Study", similar to our study, the mean serum vitamin $\mathrm{D}$ in patients with $\mathrm{OA}$ was significantly lower and the proportion of vitamin D insufficiency and deficiency was significantly higher than in controls. Vitamin D deficient men were twice as likely to have prevalent radiographic hip OA compared with controls [7].

In the "Rotterdam Study", low dietary vitamin D intake was associated with increased risk of knee OA progression over a mean follow-up time of 6.5 years [5]. In another longitudinal study of elderly women the risk of incidental hip OA increased by odds of 3.34 (95\%CI 1.13-9.86) in subjects with low serum 25-OHD levels over an average follow-up period of eight years [14]. In participants of the "Framingham Study", low serum 25-OHD predicted the development of cartilage loss and knee OA progression [14]. However, the association of vitamin D deficiency and OA was not confirmed by another longitudinal study by Felson et al. [16].

Based on current knowledge, knee OA should be considered a bone disease rather than a synovial disease [4, 8]. Early joint structural changes such as cartilage defects, loss of cartilage volume, subchondral bone expansion and bone marrow lesions are present prior to the onset
Table 2 Association between serum 25-hydroxyvitamin D (25-OHD) deficiency ${ }^{\mathrm{a}}$ and knee osteoarthritis (OA) after adjustment for gender by calculation of adjusted odds ratio (OR) and $95 \%$ confidence interval $(95 \%$ $\mathrm{CI})$ using logistic regression analysis

Serum $25-\mathrm{OHD}<20 \mathrm{ng} / \mathrm{ml}$

\begin{tabular}{llll}
\hline Patients & Independent variables & Adjusted OR $(95 \% \mathrm{CI})$ & $P$ values \\
\hline All & Serum 25-OHD $(<20$ vs. $>20 \mathrm{ng} / \mathrm{ml})$ & $1.62(0.97-2.6)$ & 0.06 \\
& Gender (female vs. male) & $3.06(1.74-5,3)$ & 0.001 \\
& Age (years) & $1.01(0.99-1.03)$ & 0.19 \\
$<60$ years & Serum 25-OHD $(<20$ vs. $>20 \mathrm{ng} / \mathrm{ml})$ & $2.26(1.15-4.4$ & 0.018 \\
& Gender (female vs. male) & $3.21(1.28-8.04)$ & 0.013 \\
$>60$ years & Serum 25-OHD $(<20$ vs. $>20 \mathrm{ng} / \mathrm{ml})$ & $1.01(0.48-5.9)$ & 0.96 \\
& Gender (female vs. male) & $2.9(1.42-5.9)$ & 0.003 \\
$<55$ years & Serum 25-OHD $(<20$ vs. $>20 \mathrm{ng} / \mathrm{ml})$ & $2.63(1.16-5.95)$ & 0.01 \\
& Gender (female vs. male) & $4.79(1.55-14.8)$ & 0.006 \\
\hline
\end{tabular}


of joint symptoms and clinical OA [18]. Bone expansion in the tibial subchondral area play an initial role in the aetiology of knee cartilage defects and cartilage loss. These defects tend to progress in symptomatic knee OA. Prevention of cartilage damage or reducing their severity by intervention may retard OA progression [18].

Inadequate levels of serum 25-OHD in patients with knee $\mathrm{OA}$ is of major concern. Since at low tissue concentrations of serum vitamin $\mathrm{D}$ bone response to pathophysiological processes is not optimal, vitamin D deficiency provides a susceptible background for OA progression [5-7, 14].

Several mechanisms such as alterations in mechanical properties of bones, increasing bone resorption by raising PTH level, increasing bone turn over, or direct effect of vitamin D metabolites on articular chondrocytes were postulated to explain the contribution of vitamin D deficiency in the progression of OA [4].

These observations provide a rationale for the measurement of serum 25-OHD in patients with OA and encourage supplementation to raise the serum concentration to adequate levels. Hence, serum 25-OHD measurement should be considered in any patients with symptoms suggestive of knee OA even before the appearance of radiographic changes. By the time radiographic $\mathrm{OA}$ is detected $10 \%$ of knee joint cartilage has already been lost [17]. Improvement of serum 25-OHD to a sufficient level augments skeletal health and alters joint response to risk factors and retards progression of OA.

A correlation of vitamin $\mathrm{D}$ with structural damage of subchondral bone has been shown [19]. There are similarities between $\mathrm{OA}$ and vitamin D deficiency with regard to increased levels of bone turnover markers [1, 7]. The potential of vitamin D treatment in suppression of turnover markers supports these observations and suggests that raising serum 25-OHD to sufficient levels may exert beneficial effects in patients with knee OA [9].

Our study has limitations which should be taken into account. Since performance of radiographic examination in asymptomatic controls was not clinically indicated, inclusion of asymptomatic $\mathrm{OA}$ to the control group cannot be ignored. This issue may lower the mean serum 25-OHD level from the actual value in the control group and reduce the mean difference between patient and control groups. Otherwise the real mean difference value between patients and controls, particularly in age groups of 60 years and over, would be greater than that observed in this study. This problem may partly explain the lack of association between vitamin D deficiency and knee OA in the age group of 60 years and older.

Patients with knee OA are expected to have higher body mass index (BMI) than controls. Obese patients may have low serum 25-OHD levels due to decreased passage of vitamin $\mathrm{D}$ from skin to the general circulation. However, higher BMI values greater than $30 \mathrm{~kg} / \mathrm{m}^{2}$ were shown to be associated with low vitamin D [20]. We did not provide data regarding BMI in this study; however, in a previous study of patients with knee OA derived from the general population of this study the mean BMI in patients with knee OA with mean age of $60 \pm 11$ years was $27.8 \pm 8.1 \mathrm{~kg} / \mathrm{m}^{2}$ [21].

We have shown an age-related modifying role for vitamin $\mathrm{D}$ deficiency which has not yet been reported. The association of serum 25-OHD deficiency with knee OA in lower age groups may be explained by greater bone health and higher activity of bone remodelling in younger than in older persons. Healthy remodelling requires the availability of adequate vitamin D [2]. Therefore, younger individuals are more dependent on vitamin $\mathrm{D}$ and are thus expected to be more sensitive to serum 25-OHD deficiency. It is unclear whether age by itself or the degree of OA which presumably is less severe in younger patients, or other factors, can explain the observed association. Comparing serum 25-OH D in patients with different stages of knee OA after allowing for age provide further information in relation to OA stage and serum 25-OHD deficiency.

The results of our study are less affected by seasonal variations, diet, and sunlight exposure or limitation of physical activities due to pain and other confounders because patients and controls of this study were drawn from the general population with unique racial and cultural backgrounds, with similar diet and sunlight exposure. Sampling in both groups was performed over the same seasonal periods and patients with disability and limitation of physical activities were excluded from the study.

In conclusion, this study has demonstrated a significant association between knee OA and vitamin D deficiency in age groups most consistent with initiation of early $\mathrm{OA}$ symptoms corresponding with the development of knee cartilage damage. Loss of knee joint cartilage, which starts at about the age of 40 years [18], progresses incrementally with age as in the normal population, and at a mean age of 57 years approximately two thirds of the joint cartilage is damaged. Identification of high risk subjects and modification of risk factors of subchondral bone such as correction of serum 25-OHD deficiency at this stage is expected to exert beneficial effects. However, the causal relationship of vitamin D deficiency in the development of cartilage defects and the potential of vitamin D treatment in the resolution of these structural lesions requires further prospective studies.

\section{References}

1. Adams JS, Hewison M (2010) Update in vitamin D. J Clin Endocrinol Metab 95:471-476

2. Holick MF (2006) High prevalence of vitamin D inadequacy and implications for health. Mayo Clin Proc 81:353-37 
3. Heidari B, Shokri Shirvani J, Firouzjahi A, Heidari P, HajianTilaki K (2010) Association between nonspecific skeletal pain and vitamin D deficiency. Int J Rheumatol 13:340-346

4. Pérez-López FR (2007) Vitamin D and its implications for musculoskeletal health in women: an update. Maturitas 58:117137

5. Bergink AP, Uitterlinden AG, Van Leeuwen JP, Buurman CJ, Hofman A, Verhaar JA, Pols HA (2009) Vitamin D status, bone mineral density, and the development of radiographic osteoarthritis of the knee: the Rotterdam Study. J Clin Rheumatol 15:230237

6. Ding C, Cicuttini F, Parameswaran V, Burgess J, Quinn S, Jones G (2009) Serum levels of vitamin D, sunlight exposure, and knee cartilage loss in older adults: the Tasmanian older adult cohort study. Arthritis Rheum 60:1381-1389

7. Chaganti RK, Parimi N, Cawthon P, Dam TL, Nevitt MC, Lane NE (2010) Association of 25-hydroxyvitamin D with prevalent osteoarthritis of the hip in elderly men: the osteoporotic fractures in men study. Arthritis Rheum 62:511-514

8. Bettica P, Cline G, Hart DJ, Meyer J, Spector TD (2002) Evidence for increased bone resorption in patients with progressive knee osteoarthritis: longitudinal results from the Chingford study. Arthritis Rheum 46:3178-3184

9. von Hurst PR, Stonehouse W, Kruger MC, Coad J (2010) Vitamin D supplementation suppresses age-induced bone turnover in older women who are vitamin D deficient. J Steroid Biochem Mol Biol 121(1-2):293-296 [Epub 2010 Mar 19 ]

10. Tsaei KS, Wahner HW, Offord KP, Melton LJ, Kumar R, Rigs BL (1987) Effect of aging on vitamin D stores and bone density in women. Calcif Tissue Int 40:241-243

11. Bischoff-Ferrari HA, Zhang Y, Kiel DP, Felson DT (2005) Positive association between serum 25-hydroxyvitamin D level and bone density in osteoarthritis. Arthritis Rheum 53:821-826

12. Mäkinen TJ, Alm JJ, Laine H, Svedström E, Aro HT (2007) The incidence of osteopenia and osteoporosis in women with hip osteoarthritis scheduled for cementless total joint replacement. Bone 40:1041-1047

13. Altman R, Asch E, Bloch D, Boil G, Borestein D, Brandt K, Cheristy W, Cooke TD, Greenwald R, Hochberg M et al (1986) Development of criteria for classification and reporting of osteoarthritis of the knee. Arthtitis Rheum 29:1039-1049

14. McAlindon TE, Felson DT, Zhang Y et al (1996) Relation of dietary intake and serum levels of vitamin D to progression of osteoarthritis of the knee among participants in the Framingham Study. Ann Intern Med 125:353-359

15. Lane NE, Gore LR, Cummings SR, Study of Osteoarthritis Fractures Research Group, et al (1999) Serum vitamin D levels and incident changes of radiographic hip osteoarthritis: a longitudinal study. Arthritis Rheum 42:854-860

16. Felson DT, Niu J, Clancy M, Aliabadi P, Sack B, Guermazi A, Hunter DJ, Amin S, Rogers G, Booth SL (2007) Low levels of vitamin $\mathrm{D}$ and worsening of knee osteoarthritis: results of two longitudinal studies. Arthritis Rheum 56:129-136

17. Jones G, Ding C, Scott F et al (2004) Early radiographic osteoarthritis is associated with substantial changes in cartilage volume and tibial bone surface area in both males and females. Osteoarthritis Cartilage 12:169-174

18. Davies-Tuck ML, Wluka AE, Wang Y, Teichtahl AJ, Jones G, Ding C, Cicuttini FM (2008) The natural history of cartilage defects in people with knee osteoarthritis. Osteoarthritis Cartilage 16:337-342

19. Dore D, Quinn S, Ding C, Winzenberg T, Jones G (2009) Correlates of subchondral BMD: a cross-sectional study. J Bone Miner Res 24:2007-2015

20. Break K, Huang WY, Fraser DR et al (2010) Low vitamin D status is associated with low physical activity, obesity, and low vitamin D intake in a large US sample of healthy middle age men and women. J Steroid Biochem Mol Biol 121:462-466

21. Heidari B, Hajian K (2004) The role of age, weight and sex in knee osteoarthritis. J Qazvin Univ Med Sci 29:10-15 